\title{
Meta
}

Journal des traducteurs

Translators' Journal

\section{CABRÉ, Maria Teresa (1993) : La terminología. Teoría, metogología, aplicaciones, traduit du catalan par Charles Tébé, Barcelona, Editorial Antártida/Empuries, 529 p.}

\section{Jean-Claude Boulanger et Monique C. Cormier}

Volume 39, numéro 3, septembre 1994

URI : https://id.erudit.org/iderudit/002110ar

DOI : https://doi.org/10.7202/002110ar

Aller au sommaire du numéro

Éditeur(s)

Les Presses de l'Université de Montréal

ISSN

0026-0452 (imprimé)

1492-1421 (numérique)

Découvrir la revue

Citer ce compte rendu

Boulanger, J.-C. \& Cormier, M. C. (1994). Compte rendu de [CABRÉ, Maria Teresa (1993) : La terminología. Teoría, metogología, aplicaciones, traduit du catalan par Charles Tébé, Barcelona, Editorial Antártida/Empuries, 529 p.] Meta, 39(3), 479-481. https://doi.org/10.7202/002110ar d'utilisation que vous pouvez consulter en ligne. 
CABRÉ, Maria Teresa (1993): La terminología. Teoría, metodología, aplicaciones, traduit du catalan par Carles Tebé, Barcelona, Editorial Antártida/Empuries, 529 p.

Un an après la publication en catalan de La terminologia. La teoria, els mètodes, les aplicacions paraît la traduction en castillan. Si Jean-Claude Corbeil signait la préface de l'édition originale, l'honneur en revient cette fois à Juan Carlos Sager.

L'ouvrage de M. T. Cabré se divise en sept chapitres, dont nous esquisserons les contenus.

Le premier chapitre (pp. 21-68) porte sur la situation et les aspects généraux de la terminologie. L'auteur présente les éléments historiques nécessaires à la compréhension de l'évolution de la discipline ainsi que les fondements qui sont à son origine. Pour ce faire, elle prend en compte les aspects sociaux et politiques, scientifiques et fonctionnels, ainsi que les aspects organisationnels, tant sur le plan national qu'international. Notons que dans la version catalane, une section était réservée à la terminologie en Catalogne. Dans la version castillane, on a préféré donner un aperçu de la terminologie en Amérique latine. Ce chapitre constitue une excellente introduction à l'histoire de la terminologie.

Le deuxième chapitre (pp. 69-122), qui comprend cinq parties, s'intéresse au caractère interdisciplinaire de la terminologie. $M$. T. Cabré traite successivement des rapports entre la terminologie et la linguistique, les sciences cognitives, la communication, la documentation et enfin l'informatique. Elle prend position sur la place de la terminologie dans les sciences du langage et montre clairement que celle-ci fait partie de la linguistique, qu'elle n'est pas une forme de lexicologie appliquée, pas plus qu'elle n'est à la remorque de la traduction. 
Les fondements linguistiques de la terminologie font l'objet du troisième chapitre (pp. 123-259), le plus important de l'ouvrage. Dans ce chapitre, l'auteur se penche de façon approfondie sur le concept de «langue de spécialité», sur l'unité terminologique ainsi que sur la documentation spécialisée. Elle situe d'abord les langues de spécialité dans la chaîne linguistique, du phonème au texte, puis elle en détaille les caractéristiques. Elle présente ensuite un certain nombre de définitions proposées par d'autres chercheurs, notamment R. Kocourek, L. Hoffmann, G. Rondeau, A. Rey, B. Quemada, définitions qu'elle commente et critique. M. T. Cabré propose ensuite sa propre définition de langue de spécialité: «... subconjuntos del lenguaje general caracterizados pragmáticamente por tres variables: la temática, los usuarios y las situaciones de comunicación» (p. 139). La partie qui suit est consacrée au terme. Y sont étudiés en profondeur la dénomination, le concept et les relations entre dénomination et concept. Enfin, le chapitre se termine sur la documentation spécialisée. L'auteur propose une typologie de la documentation, puis traite des étapes du travail documentaire. Sont abordées en dernier lieu les banques documentaires et l'utilisation de la documentation par le terminologue.

Le quatrième chapitre (pp. 261-352) porte sur la pratique de la terminologie : la terminographie. Il se divise en trois parties : les fondements de la praxis, les supports de travail et les méthodes de travail. La fiche est expliquée de façon complète et les méthodes utilisées tant en recherche thématique qu'en recherche ponctuelle sont étudiées dans le détail.

La terminotique est examinée dans le chapitre cinq (pp. 353-422). Quatre dimensions sont ici envisagées: le concept même de «terminotique», les apports de l'informatique à la terminologie, les industries de la langue, les banques de données et les banques de termes.

C'est dans le sixième chapitre (pp. 423-460) que M.T. Cabré aborde le domaine de la normalisation en général et présente les principaux organismes de normalisation. Il est ensuite question de la normalisation terminologique, qui touche à la fois les termes ainsi que les principes et méthodes. C'est dans ce chapitre que la néologie est l'objet de l'attention de l'auteur.

Le septième et dernier chapitre (pp. 461-481) est consacré à l'aspect professionnel de la terminologie. On y scrute le rôle du terminologue dans un service linguistique. Y sont également tissés les liens entre la terminologie comme discipline de la linguistique et le milieu professionnel. L'auteur termine le chapitre en discutant la formation des terminologues. Elle insiste surtout sur la nécessité d'une double formation en linguistique et en terminologie.

L'ouvrage s'achève par une série d'annexes dans lesquelles sont présentés, entre autres, des exemples de fiches. Ces annexes sont suivies d'une importante bibliographie.

La version castillane offre les mêmes qualités didactiques que la version originale en catalan: un texte clair, dans une traduction bien enlevée, sans obscurité ni ambiguïté, un format pratique, une typographie agréable, une mise en pages aérée ainsi qu'une abondance d'intertitres qui facilitent la lecture. Malheureusement, si la plupart des coquilles qui parsemaient de nombreuses citations en d'autres langues, notamment en français et en anglais, ont été corrigées dans cette version castillane, de nombreuses erreurs de retranscription ont subsisté.

On saura gré à l'auteur d'avoir rédigé une synthèse exhaustive et à jour des connaissances sur le domaine, d'avoir analysé la terminologie comme une discipline indépendante, comme une composante à part entière des sciences du langage, et d'avoir su se garder de l'assujettir à l'activité traductionnelle, comme on le fait trop souvent dans certains milieux. La terminologie est beaucoup plus en effet qu'un simple satellite de la traduction. Il faut recommander la lecture de cet ouvrage, non seulement parce qu'il se veut l'ouvrage le plus complet à l'heure actuelle sur la terminologie, mais également parce qu'il repré- 
sente le livre de références sur les rapports entre cette discipline et la pratique linguistique, la pratique cognitive et la pratique sociale.

JEAN-CLAUDE BOULANGER

Université Laval, Québec, Canada

MONIQUE C. CORMIER

Université de Montréal, Montréal, Canada 